\title{
Effect of the cyst fluid concentration of iron on infertility in patients with ovarian endometrioma
}

\author{
MIKA NAGAYASU $^{1}$, SHOGO IMANAKA ${ }^{1,2}$, MAI KIMURA ${ }^{1}$, \\ SACHIYO MARUYAMA $^{1}$, NAOKI KAWAHARA ${ }^{1}$ and HIROSHI KOBAYASHI ${ }^{1,2}$ \\ ${ }^{1}$ Department of Obstetrics and Gynecology, Nara Medical University, Kashihara, \\ Nara 634-8522; ${ }^{2}$ Ms. Clinic MayOne, Kashihara, Nara 634-0813, Japan
}

Received July 19, 2020; Accepted August 31, 2020

DOI: $10.3892 /$ wasj.2020.66

\begin{abstract}
The causes of infertility in women with endometriosis may range from anatomical distortions to various pathophysiological disturbances. The aim of the present study was to examine the effects of the cyst fluid (CF) concentration of iron on infertility in patients with ovarian endometrioma (OMA). Patients with histologically confirmed OMA were enrolled at the Department of Obstetrics and Gynecology, Nara Medical University Hospital between 2013 and 2019. The patients were divided into 2 groups, namely women experiencing current infertility (infertile group) and those without complaints of infertility (non-infertile group). There were 2 types of patients in the infertile group: Patients who failed to achieve a clinical pregnancy following $\geq 12$ months of regular unprotected sexual intercourse and those who had already been treated at fertility clinics. The CF concentration of iron was measured by the inductively coupled plasma-optical emission spectrometry (ICP-OES) method. The clinical data were analyzed retrospectively. A total of 77 patients were enrolled in the present study. Among these, 32 (41.6\%) patients had infertility. When compared with the non-infertile women, the infertile women were significantly younger (median age, 35 years; range, $24-47$ years; vs. median age, 40 years; range, $21-53$ years; respectively; $\mathrm{P}=0.003)$. The $\mathrm{CF}$ concentrations of iron (median, $324.8 \mathrm{mg} / \mathrm{l}$; range, $71.3-1046.3 \mathrm{mg} / \mathrm{l}$; vs. median, $226.5 \mathrm{mg} / 1$; range, $65.3-737.5 \mathrm{mg} / 1$; respectively; $\mathrm{P}=0.019$ ) were significantly higher in the infertile group compared with the non-infertile group. Multivariate logistic regression analysis indicated that age at diagnosis ( $\leq 38$ years), the CF concentrations of iron $(>326.6 \mathrm{mg} / \mathrm{l})$ and the infertility index (iron/age ratio, >8.37) were independent risk factors for endometriosis-related infertility. Multivariate analysis revealed that age (HR, 6.44; 95\% CI, 2.06-20.12) and iron (HR, 4.90; 95\% CI,
\end{abstract}

Correspondence to: Professor Hiroshi Kobayashi, Department of Obstetrics and Gynecology, Nara Medical University, 840 Shijo-cho, Kashihara, Nara 634-8522, Japan

E-mail: hirokoba@naramed-u.ac.jp

Key words: ovarian endometrioma, infertility, iron, oxidative stress
1.48-16.22) were independent factors for the identification of patients with OMA who presented with a complaint of infertility. In addition, the infertility index (iron/age ratio, $>8.37$; HR, 4.85; 95\% CI, 1.01-23.27) was an important predictor of infertility. ROC curve analysis also revealed that the areas under the ROC (AUC) for age, iron and infertility index were $0.699,0.666$ and 0.731 , respectively. On the whole, the findings of the present study demonstrate that age at diagnosis and the CF concentration of iron may be potentially effective markers for predicting infertility in women with OMA.

\section{Introduction}

Endometriosis is a common, estrogen-dependent inflammatory disease characterized by the presence of endometrial tissue outside the uterine cavity (1). Endometriosis includes superficial peritoneal disease, ovarian endometrioma (OMA) and deep endometriosis (DE) lesions as major phenotypes. The main symptoms include pelvic pain, such as dysmenorrhea, dyspareunia and chronic pelvic pain, and infertility. Statistically, 30-50\% of females with endometriosis experience infertility (2). Endometriosis-related pain symptoms may be associated with the severity of diseases, such as adhesions and posterior cul-de-sac and uterosacral lesions (3). Anatomical abnormalities with adhesions and fibrosis lead to infertility, although even mild endometriosis can cause infertility (4). The severity of symptoms, including pain and infertility, is not always associated with the extent of endometriosis.

Thus far, non-invasive diagnostic approaches, such as transvaginal ultrasonography (TVS), magnetic resonance imaging (MRI) and blood tests have exhibited considerable power in the diagnosis of endometriosis (5). TVS and MRI can help visualize OMA and DE, but not superficial peritoneal disease. In addition, imaging is inadequate for the prediction of endometriosis-associated infertility. Therefore, the lack of non-invasive diagnostic tests that can predict infertility leads to the long delay before treatment (6). The association between endometriosis and infertility is well known; however, the mechanisms underlying endometriosis-associated infertility have not yet been well established. It has been pointed out that inflammatory cytokines and oxidative stress may adversely affect eggs, sperm and fertilized eggs, leading to impaired fertilization and implantation, and decreased fertility $(7,8)$. 
Recently, Yoshimoto et al reported that the cyst fluid (CF) concentrations of iron were elevated in patients with OMA (9). Excess labile and bioactive iron concentrations can enhance toxic radical generation and induce oxidative stress (10). A preclinical study revealed that the CF concentration of iron was associated with oxidative stress, and was involved in endometriosis progression and malignant transformation (11). The present study measured iron levels to assess whether iron-dependent oxidative stress is involved in infertility, as it has previously been demonstrated that oxidative stress is associated with infertility in endometriosis (12).

The aim of the present study was to evaluate the effects of the CF concentration of iron on infertility in patients with OMA. The discovery of a sensitive, specific and non-invasive biomarker for the prediction of endometriosis-associated infertility may hold promise for the development of earlier diagnosis and treatment strategies.

\section{Materials and methods}

Patients and study design. The present study was a single-center, retrospective cohort study aimed at investigating the association between infertility and the CF concentration of iron in patients with OMA. Newly diagnosed patients were registered consecutively from February, 2013 to May, 2019 at the Department of Obstetrics and Gynecology, Nara Medical University Hospital, Kashihara, Japan. Patients who visited the hospital complained of pelvic pain and/or infertility. Participants were recommended to undergo routine TVS and pre-operative MRI, performed as part of the clinical care program. A sonography was performed by 2 experienced operators (MK and SM) with a single ultrasound system (Voluson E8; GE Healthcare) using a transvaginal transducer (5-7.5 MHz). MRI was obtained on a 3T system using T1W and T2W sequences (Magnetom Verio, Siemens Healthcare). The TVS test and MRI were performed within 4 weeks before surgery.

The patients were divided into 2 groups, namely women experiencing current infertility (infertile group) and those without complaints of infertility (non-infertile group). There were 2 types of patients in the infertility group: Patients who failed to achieve a clinical pregnancy following $\geq 12$ months of regular unprotected sexual intercourse (13) and those who have already been treated at fertility hospitals. Patients with OMA who received laparotomy or laparoscopic surgery were enrolled in the present study. Surgery was performed not only to treat infertility, but also to improve pain. A histological diagnosis was confirmed by surgical pathology. Exclusion criteria were any of the following: No pathology, benign ovarian tumors other than OMA, OMA co-existing with DIE, adenomyosis and malignant transformation, prior ovarian surgery, unmarried women, women who did not wish to become pregnant, menopausal women and women who had received hormonal therapy within 3 months. A form of regional medical cooperation was established and maintained with fertility hospitals, in order for all potential patients to be referred to the appropriate reproductive specialist following discharge. Therefore, detailed information on the variables involved in the demographic and clinical characteristics, such as the length of infertility, ovarian function, semen status, or comorbidities of the infertile women could not be obtained. The present study was conducted under the guidelines that had been approved by the Medical Ethics Committee of Nara Medical University. Written informed consent was obtained from each patient.

Quantification of iron concentrations in CF. Cyst fluid samples were obtained during surgery. Cyst fluids harvested were collected into a plastic tube without an anticoagulant and centrifuged at $2,000 \times \mathrm{g}$ for $10 \mathrm{~min}$ at $4^{\circ} \mathrm{C}$. Supernatants were immediately aliquoted and kept frozen at $-20^{\circ} \mathrm{C}$ within $1 \mathrm{~h}$ of collection until the time of analysis. The amount of iron was determined by inductively coupled plasma optical emission spectrometry (ICP-OES) (Vista MPX, Varian, Inc.) with internal standard method as described previously (9). The results of the assays were not available to the clinicians and therefore did not influence subsequent patient management.

Statistical analysis. SPSS 25.0 (SPSS Inc.) statistical software was used for the statistical analysis. Comparison of categorical variables between groups was performed using the Chi-squared test. The Mann-Whitney U-test was used for the univariate analysis of numerical data. The cut-off value of cyst fluid concentrations of iron was calculated with the highest sensitivity and specificity for predicting the two groups based on the receiver operator characteristic (ROC) curve analysis. The potential factors for predicting infertility were determined by logistic regression analysis. The independent factors were identified by multivariate regression analysis. Hazard ratios (HRs) were calculated using a two-stage regression estimate with the infertility as the instrumental variable to examine associations with age and iron (Fig. 1) or parity and infertility index (Fig. 2) by logistic regression and Cox regression, respectively. Furthermore, the infertility index, defined as iron level/age, was also subjected to a multivariate analysis. Differences with $\mathrm{P}<0.05$ were considered to be statistically significant.

\section{Results}

The present study included 77 patients who met the inclusion and exclusion criteria during the period of the study. Among these, 32 (41.6\%) patients had infertility. Two women in the infertile group became pregnant after receiving the assisted reproductive technique (ART). The non-infertile group included 36 women who have previously given birth due to one or more spontaneous pregnancies. In addition, 9 women in the non-infertile group had one or more miscarriages, but failed to have live births. The patient demographic factors and clinical characteristics of the study population are summarized in Table I. The patients were significantly younger in the infertile group than in the non-infertile group (median age, 35 years; range, 24-47 years; vs. median age, 40 years; range, $21-53$ years, respectively; $\mathrm{P}=0.003$ ). Since the 2 groups of patients were not homogeneous, with a median age of 35 and 40 years for the infertile and non-infertile groups, respectively, the association between age and the $\mathrm{CF}$ concentrations of iron was analyzed. In the analyses of data from all the study subjects, no significant correlation between patient age and the CF concentration of iron was observed 
A

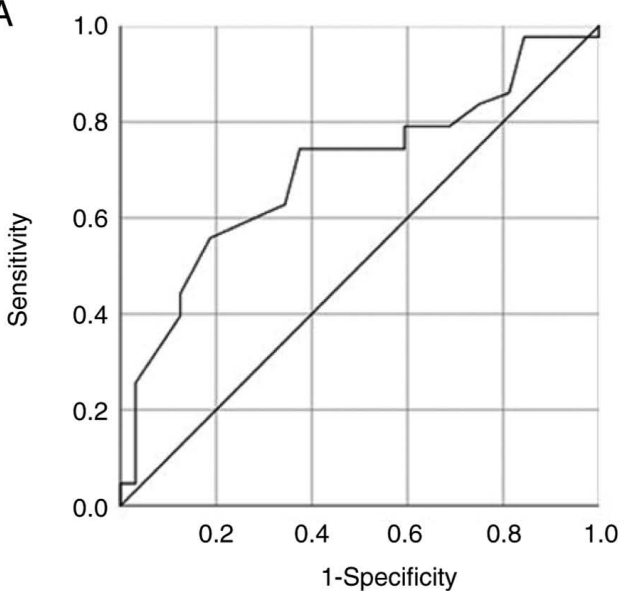

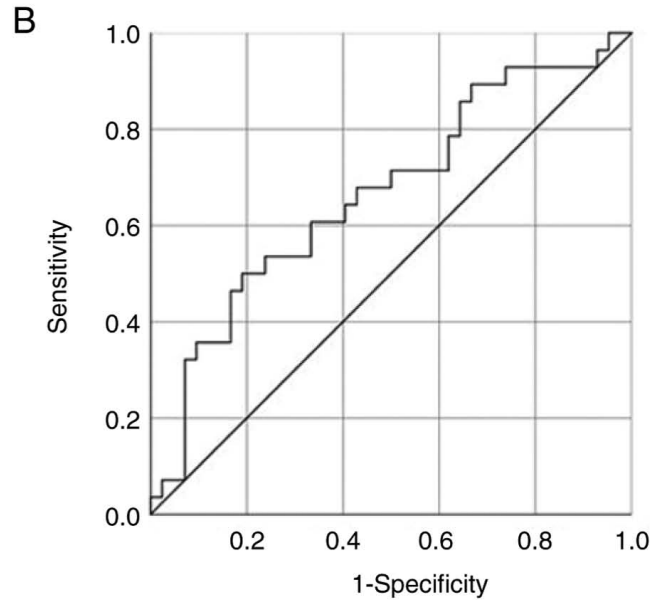

Figure 1. ROC curves with calculated AUCs of 2 indicators: (A) Age at diagnosis and (B) CF concentration of iron. The ROC curve was derived from the sensitivity and the specificity. ROC, receiving operating characteristic; AUC, area under the curve; CF, cyst fluid.

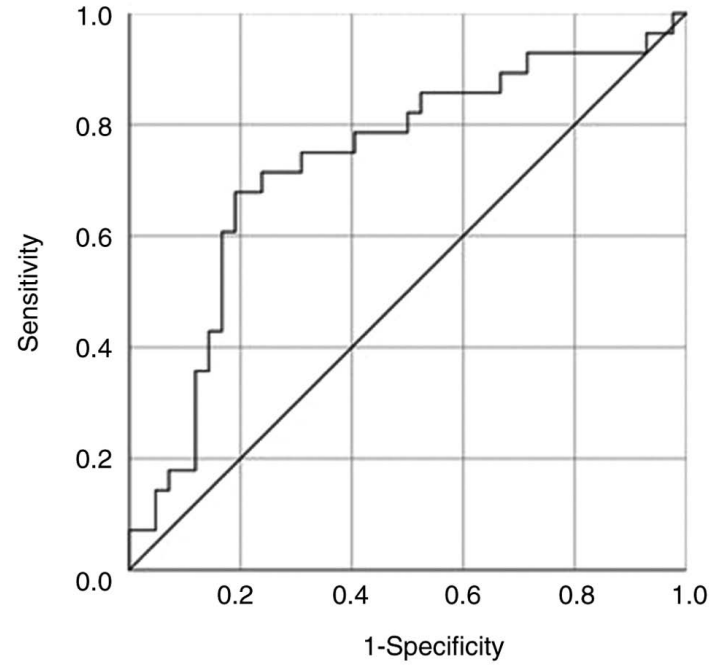

Figure 2. A ROC curve with a calculated AUC of the infertility index. The ROC curve derived from the sensitivity and the specificity. ROC, receiving operating characteristic; AUC, area under the curve.

[y (CF concentration of iron) $=-1.63 x($ age $\left.)+343.0, \mathrm{r}^{2}=0.004\right]$ (data not shown). Not surprisingly, the infertile group exhibited a significantly lower parity compared to the non-infertile group $(\mathrm{P}<0.001)$. There were no significant differences among the 2 groups in variables, such as pre-operative CA125 levels, pre-operative CA19-9 levels, cyst diameter and tumor localization. The $\mathrm{CF}$ concentrations of iron were significantly higher in the infertile group compared with the non-infertile group (median, $324.8 \mathrm{mg} / \mathrm{l}$; range, $71.3-1046.3 \mathrm{mg} / \mathrm{l}$; vs. median, 226.5; range, 65.3-737.5, respectively; $\mathrm{P}=0.019$ ) (Table I).

Since parity is the most important factor that reflects the outcome of infertility, the addition of parity to the multivariate analysis eliminated the other 2 variables (age and the $\mathrm{CF}$ concentration of iron). First, the present study examined whether age at diagnosis and the $\mathrm{CF}$ concentration of iron could identify women experiencing current infertility (infertile group). ROC curves were applied to assess the potential utility of these indicators in discriminating between the infertile and non-infertile groups (Fig. 1). ROC curve analysis discriminated the infertile group from the non-infertile group with an AUC value of 0.699 and an optimal cut-off value of 37.5 years of age (sensitivity, $74.4 \%$; specificity, $62.5 \%$ ) (Fig. 1A). It was also found that the $\mathrm{CF}$ iron measurement successfully discriminated between the 2 groups (AUC, 0.666 ) with an optimal cut-off value of $326.6 \mathrm{mg} / \mathrm{l}$ (sensitivity, 50.0\%; specificity, $81.0 \%$ ) (Fig. 1B). Based on the above-mentioned data, a multivariate logistic regression analysis was conducted to identify independent variables associated with infertility (Table II). The results revealed that age at diagnosis (HR, 6.44; 95\% CI, 2.06-20.12) and the CF concentration of iron (HR, 4.90; 95\% CI, 1.48-16.22) were able to independently identify patients with OMA experiencing current infertility.

Second, an age $<37.5$ years and a CF concentration of iron $>326.6 \mathrm{mg} / 1$ were predictors of infertility (Table II); thus, a combined analysis of the 2 variables was performed. The iron level/age ratio was defined as an infertility index. ROC curve analysis discriminated between the infertile group from the non-infertile group with an optimal iron level/age ratio cut-off value of 8.37 with an AUC value of 0.731 (sensitivity, 67.9\%; specificity, $81.0 \%$ ) (Fig. 2). Multivariate logistic regression analysis revealed that both parity (HR, 0.012; 95\% CI, 0.001-0.10; $\mathrm{P}<0.001)$ and infertility index (HR, 4.85; $95 \% \mathrm{CI}, 1.01-23.27 ; \mathrm{P}=0.049$ ) significantly predicted infertility (Table III).

\section{Discussion}

In order to examine the role of iron in infertile women, the present study analyzed the pre-operative CF concentrations of iron in 77 patients with OMA who underwent laparoscopic surgery. Women experiencing current infertility were 5 years younger than those without complaints of infertility (median age, 35 vs. 40 years). The present study demonstrated for the first time, at least to the best of our knowledge, that the CF concentrations of total iron were significantly higher in infertile patients than in non-infertile women (median, $324.8 \mathrm{mg} / \mathrm{l}$ vs. $226.5 \mathrm{mg} / \mathrm{l} ; \mathrm{P}=0.019)$. When the $\mathrm{CF}$ concentration of iron was $\geq 326.6 \mathrm{mg} / \mathrm{l}$, the patient was considered to be infertile, with a sensitivity and specificity of 50.0 and $81.0 \%$, respectively. In addition, the combination of 2 variables (infertility 
Table I. Demographic and clinical characteristics of patients in the infertile and non-infertile groups.

\begin{tabular}{|c|c|c|c|}
\hline & Non-infertile group $(n=45)$ & Infertile group $(n=32)$ & P-value \\
\hline Age, years; median (range) & $40(21-53)$ & $35(24-47)$ & $0.003^{\mathrm{a}}$ \\
\hline \multicolumn{4}{|l|}{ Parity } \\
\hline 0 & 9 & 30 & \\
\hline$\geq 1$ & 36 & 2 & $<0.001^{\mathrm{b}}$ \\
\hline CA125; median (range) & $65.5(10.0-1504.0)$ & $58.0(16.0-1830.0)$ & $0.519^{\mathrm{a}}$ \\
\hline CA19-9; median (range) & $25.0(1.0-252.0)$ & $27.5(1.0-380.0)$ & $0.783^{\mathrm{a}}$ \\
\hline Tumor diameter; median (range) & $65.0(27.0-193.0)$ & $70.0(39.0-142.0)$ & $0.103^{\mathrm{a}}$ \\
\hline \multicolumn{4}{|l|}{ Localization } \\
\hline Unilateral & 28 & 26 & \\
\hline Bilateral & 15 & 6 & $0.124^{\mathrm{a}}$ \\
\hline CF concentration of iron; median (range) & $226.5(65.3-737.5)$ & $324.8(71.3-1046.3)$ & $0.019^{\mathrm{a}}$ \\
\hline
\end{tabular}

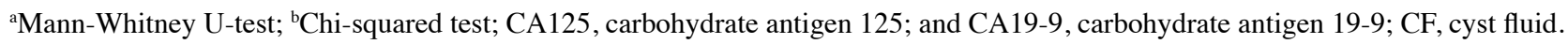

Table II. The univariate and multivariate logistic regression analysis for identifying women experiencing current infertility.

\begin{tabular}{|c|c|c|c|c|}
\hline \multirow[b]{3}{*}{ Variables } & \multicolumn{4}{|c|}{ Infertility } \\
\hline & \multicolumn{2}{|c|}{ Univariate analysis } & \multicolumn{2}{|c|}{ Multivariate analysis } \\
\hline & $\operatorname{HR}(95 \% \mathrm{CI})$ & $\mathrm{P}$-value & $\operatorname{HR}(95 \% \mathrm{CI})$ & P-value \\
\hline \multicolumn{5}{|c|}{ Age, years } \\
\hline$\geq 38$ & 1 & & & \\
\hline$<38$ & $5.03(1.83-13.80)$ & 0.002 & $6.44(2.06-20.12)$ & 0.001 \\
\hline \multicolumn{5}{|c|}{ CF concentration of iron } \\
\hline$\leq 326.6$ & 1 & & & \\
\hline$>326.6$ & $4.25(1.46-12.37)$ & 0.008 & $4.90(1.48-16.22)$ & 0.009 \\
\hline
\end{tabular}

CF, cyst fluid.

Table III. Univariate and multivariate logistic regression analysis for the identification of women experiencing current infertility.

\begin{tabular}{|c|c|c|c|c|}
\hline \multirow[b]{3}{*}{ Variables } & \multicolumn{4}{|c|}{ Infertility } \\
\hline & \multicolumn{2}{|c|}{ Univariate analysis } & \multicolumn{2}{|c|}{ Multivariate analysis } \\
\hline & HR (95\% CI) & P-value & $\mathrm{HR}(95 \% \mathrm{CI})$ & P-value \\
\hline \multicolumn{5}{|l|}{ Parity } \\
\hline 0 & 1 & & 1 & \\
\hline$\geq 1$ & $0.018(0.004-0.09)$ & $<0.001$ & $0.012(0.001-0.10)$ & $<0.001$ \\
\hline \multicolumn{5}{|c|}{ Infertility index } \\
\hline$\leq 8.37$ & 1 & & 1 & \\
\hline$>8.37$ & $8.97(2.97-27.10)$ & $<0.001$ & $4.85(1.01-23.27)$ & 0.049 \\
\hline
\end{tabular}

index; iron level/age ratio) exhibited a high sensitivity $(67.9 \%)$ and specificity $(81.0 \%)$ in predicting current infertility in patients with OMA.

First, the severity of endometriosis is dependent on anatomical factors and patient background. Age, the duration of infertility, body mass index, the duration of the menstrual cycle, history of abortion, dyspareunia, pelvic pain and a family history of endometriosis are risk factors, and some may be independent predictors of infertility associated with endometriosis (14). In addition as regards various risk factors, 
some classifications have been reported to predict the severity of endometriosis and infertility (15). Endometriosis has often been classified by the size of its anatomical lesions; however, not only the size of the lesions, but the location is also important (15). The endometriosis fertility index (EFI) has more predictive power for fecundity, in vitro fertilization (IVF) outcomes, or post-operative pregnancy in patients with endometriosis revised American than the Fertility Society classification (r-AFS classification) (16-20).

Furthermore, endometriosis and its severity have been reported to be dependent on multiple biochemical, genetic and environmental factors, such as hormonal factors, altered immune system, inflammation, growth factors, an imbalance between pro-apoptosis and anti-apoptosis, increased neuroangiogenesis, familial predisposition, genetic alterations, diet, environmental factors and excessive oxidative stress (21-23). Redox-related changes in the peritoneal microenvironment are closely associated with the pathogenesis of endometriosis, creating favorable conditions for endometriotic cell proliferation and survival. A number of researchers have investigated factors associated with the disease severity using surgically resected tissue, follicular fluid, peritoneal fluid, and blood. Wang et al reported that inflammatory factors, such as interleukin (IL)-6, IL-10, IL-13 and tumor necrosis factor (TNF)- $\alpha$, could be indicators for the diagnosis of endometriosis with infertility (24). Cyclooxygenase-2 (COX-2), a rate-limiting enzyme of prostaglandin $(\mathrm{PG})$ synthesis, plays a crucial role in the inflammation, proliferation and spread of endometriotic lesions through the upregulation of certain growth factors, such as transforming growth factor (TGF)- $\beta$ expression (25). In the peritoneal fluid of infertile women with endometriosis, increased concentrations of PGs have been shown to cause adverse effects on fertilization, implantation and embryonic growth, which will lead to infertility (26). Furthermore, an imbalance between reactive oxygen species (ROS) and the antioxidant system in the follicular fluid causes abnormal oocyte development and poor egg quality through DNA, cytoskeleton and cell membrane damage (27). Excessive oxidative stress is regarded as a possible mechanism of endometriosis-related infertility (27). Inflammatory cytokines, ILs and oxidative stress may all be reliable markers for diagnosing endometriosis and its severity (12). However, the methods for non-invasively predicting or diagnosing infertility associated with endometriosis are extremely limited, and no clinically available markers have been reported thus far, at least to the best of our knowledge.

The present study found that age at diagnosis and the $\mathrm{CF}$ concentrations of iron could predict the risk of infertility in patients with OMA. It is considered that women who presented with complaints of infertility are more likely to be younger than women without infertility as they often visit the clinic for infertility counseling. The identification of risk factors can help select populations that are prone to infertility.

Second, the present study aimed to determine whether iron can induce infertility. When red blood cells are hemolyzed in the peritoneal cavity or endometriotic cysts, hemoglobin releases heme iron and free iron (28). Hemoglobin undergoes autoxidation of the iron in its heme groups and produces superoxide radicals through conversion to methemoglobin (10). Bioactive free iron also generates hydroxyl radicals, a potent ROS, through the Fenton reaction (10). ROS, such as superoxide anion, hydroxyl radical and hydrogen peroxide, are inflammatory mediators known to exert deleterious effects by causing DNA damage, methylation and epigenetic errors (29,30). Therefore, iron-induced oxidative stress adversely affects female and male gametes, sperm fertilizing ability, implantation, embryo development, uterine receptivity, ART outcome and pregnancy rates after IVF, resulting in infertility (12). Based on the above, it was thus speculated that women with a high CF concentration of iron may be more likely to become infertile. Women who wished to become pregnant may require accurate counseling and an appropriate conception plan, depending on iron levels.

Third, over the past decade, certain non-invasive methods have been developed to quantify iron concentrations in human organs. There are at least 2 different techniques for the quantification of the iron concentration: T2 magnetic resonance relaxometry methods and near infrared optical method. The hepatic and cardiac iron content can be estimated on the effective transverse relaxation rate (R2*) (31). Recently, T2 relaxometry has allowed the non-invasive quantification of iron levels in CF and has enabled the diagnosis of endometriosis (32). In addition, near infrared spectroscopy is a validated method that allows for the quantification of the CF concentration of iron non-invasively and repeatedly (11). A new device, consisting of transvaginal ultrasonography and near infrared spectroscopy system (composite-type optical device), is currently under development for the non-invasive quantification of iron concentration in endometriotic CF $(11,33)$. Iron quantification may assist in the identification of patients at high risk of infertility.

Finally, there are some limitations to the present study. Patients with adenomyosis and/or DIE were excluded from the study to evaluate the effects of OMA itself on infertility. As a result, the small sample size limits the power of the present study. It is necessary to accumulate data on CF concentrations of iron in a large number of patients for clinical application. Moreover, the cause of infertility was not clarified in the present study. In addition, the present study does not mention that the $\mathrm{CF}$ concentration of iron is a predictor of post-operative infertility.

In conclusion, the present study demonstrated that patients with OMA experiencing current infertility visited the Nara Medical University Hospital at a younger age than those without complaints of infertility, and that the CF concentration of iron was significantly higher in the infertile group than in the non-infertile group. If the CF concentration of iron is $\geq 326.6 \mathrm{mg} / \mathrm{l}$ or the iron/age ratio is $\geq 8.37$, the cause of infertility should be evaluated for women who wish to become pregnant. CF iron may provide useful information for comprehensive counseling and treatment decisions regarding endometriosis-related infertility. Future studies are required however, to elucidate the key mechanisms that link iron with infertility.

\section{Acknowledgements}

Not applicable. 


\section{Funding}

The present was supported by JSPS KAKENHI (grant nos. 20K09604, 20K09647 and 20K09648).

\section{Availability of data and materials}

The datasets used and/or analyzed during the current study are available from the corresponding author on reasonable request.

\section{Authors' contributions}

SM, SI, MK and MN performed the literature search and collected data using the PubMed database. $\mathrm{MN}$ and $\mathrm{HK}$ made substantial contribution to the conception of the study. SI contributed to the study design and interpretation of the included research studies. NK performed the statistical analysis. The final version of the manuscript has been read and approved by all authors.

\section{Ethics approval and consent to participate}

The present study was conducted under the guidelines that had been approved by the medical ethics committee of the Nara Medical University. Written informed consent was obtained from each patient.

\section{Patient consent for publication}

Not applicable.

\section{Competing interests}

The authors declare that they have no competing interests.

\section{References}

1. Koninckx PR, Ussia A, Adamyan L, Wattiez A, Gomel V and Martin DC: Pathogenesis of endometriosis: The genetic/epigenetic theory. Fertil Steril 111: 327-340, 2019.

2. Meuleman C, Vandenabeele B, Fieuws S, Spiessens C, Timmerman D and D'Hooghe T: High prevalence of endometriosis in infertile women with normal ovulation and normospermic partners. Fertil Steril 92: 68-74, 2009.

3. Hsu AL, Sinaii N, Segars J, Nieman LK and Stratton P: Relating pelvic pain location to surgical findings of endometriosis. Obstet Gynecol 118: 223-230, 2011.

4. Tamburro S, Canis M, Albuisson E, Dechelotte P, Darcha C and Mage G: Expression of transforming growth factor betal in nerve fibers is related to dysmenorrhea and laparoscopic appearance of endometriotic implants. Fertil Steril 80: 1131-1136, 2003.

5. Nisenblat V, Bossuyt PM, Farquhar C, Johnson N and Hull ML: Imaging modalities for the non-invasive diagnosis of endometriosis. Cochrane Database Syst Rev 2: CD009591, 2016.

6. Agarwal SK, Chapron C, Giudice LC, Laufer MR, Leyland N, Missmer SA, Singh SS and Taylor HS: Clinical diagnosis of endometriosis: A call to action. Am J Obstet Gynecol 220: 354.e1-354.e12, 2019.

7. Sharma I, Dhaliwal LK, Saha SC, Sangwan S and Dhawan V: Role of 8-iso-prostaglandin F2alpha and 25-hydroxycholesterol in the pathophysiology of endometriosis. Fertil Steril 94: 63-70, 2010.

8. Choi YS, Cho S, Seo SK, Park JH, Kim SH and Lee BS: Alteration in the intrafollicular thiol-redox system in infertile women with endometriosis. Reproduction 149: 155-162, 2015.
9. Yoshimoto C, Iwabuchi T, Shigetomi H and Kobayashi H: Cyst fluid iron-related compounds as useful markers to distinguish malignant transformation from benign endometriotic cysts. Cancer Biomark 15: 493-499, 2015.

10. Iwabuchi T, Yoshimoto C, Shigetomi H and Kobayashi H: Oxidative stress and antioxidant defense in endometriosis and its malignant transformation. Oxid Med Cell Longev 2015: 848595, 2015 .

11. Kobayashi H, Yamada Y, Kawahara N, Ogawa K and Yoshimoto C: Modern approaches to noninvasive diagnosis of malignant transformation of endometriosis. Oncol Lett 17: 1196-1202, 2019.

12. Gupta S, Goldberg JM, Aziz N, Goldberg E, Krajcir N and Agarwal A: Pathogenic mechanisms in endometriosis-associated infertility. Fertil Steril 90: 247-257, 2008.

13. Zegers-Hochschild F, Adamson GD, de Mouzon J, Ishihara O, Mansour R, Nygren K, Sullivan E, Vanderpoel S; International Committee for Monitoring Assisted Reproductive Technology and World Health Organization: International Committee for monitoring assisted reproductive technology (ICMART) and the World Health Organization (WHO) revised glossary of ART terminology, 2009. Fertil Steril 92: 1520-1524, 2009.

14. Ashrafi M, Sadatmahalleh SJ, Akhoond MR and Talebi M: Evaluation of risk factors associated with endometriosis in infertile women. Int J Fertil Steril 10: 11-21, 2016.

15. Bouquet de Joliniere J, Major A, Ayoubi JM, Cabry R, Khomsi F, Lesec G, Frydman R and Feki A: It is necessary to purpose an add-on to the american classification of endometriosis? This disease can be compared to a malignant proliferation while remaining benign in most cases. Endogram ${ }^{\circledR}$ is a new profile witness of its evolutionary potential. Front Surg 6: 27, 2019.

16. Zhou Y, Lin L, Chen Z, Wang Y, Chen C, Li E and Wu R: Fertility performance and the predictive value of the endometriosis fertility index staging system in women with recurrent endometriosis: A retrospective study. Medicine (Baltimore) 98: e16965, 2019.

17. Negi N, Roy KK, Kumar S, Nair VG and Vanamail P: Clinical outcome analysis and correlation of reproductive outcome with endometriosis fertility index in laparoscopically managed endometriosis patients: A retrospective cohort study. J Hum Reprod Sci 12: 98-103, 2019.

18. Li X, Zeng C, Zhou YF, Yang HX, Shang J, Zhu SN and Xue Q: Endometriosis fertility index for predicting pregnancy after endometriosis surgery. Chin Med J (Engl) 130: 1932-1937, 2017.

19. Wang W, Li R, Fang T, Huang L, Ouyang N, Wang L, Zhang Q and Yang D: Endometriosis fertility index score maybe more accurate for predicting the outcomes of in vitro fertilisation than $\mathrm{r}$-AFS classification in women with endometriosis. Reprod Biol Endocrinol 11: 112, 2013.

20. Revised American society for reproductive medicine classification of endometriosis: 1996. Fertil Steril 67: 817-821, 1997.

21. Tosti C, Pinzauti S, Santulli P, Chapron C and Petraglia F: Pathogenetic mechanisms of deep infiltrating endometriosis. Reprod Sci 22: 1053-1059, 2015.

22. Ahn SH, Monsanto SP, Miller C, Singh SS, Thomas R and Tayade C: Pathophysiology and immune dysfunction in endometriosis. Biomed Res Int 2015: 795976, 2015.

23. McKinnon B, Mueller M and Montgomery G: Progesterone resistance in endometriosis: An acquired property? Trends Endocrinol Metab 29: 535-548, 2018.

24. Wang XM, Ma ZY and Song N: Inflammatory cytokines IL-6, IL-10, IL-13, TNF- $\alpha$ and peritoneal fluid flora were associated with infertility in patients with endometriosis. Eur Rev Med Pharmacol Sci 22: 2513-2518, 2018.

25. Yuan L, Shen F, Lu Y, Liu X and Guo SW: Cyclooxygenase-2 overexpression in ovarian endometriomas is associated with higher risk of recurrence. Fertil Steril 91 (Suppl 4): S1303-S1306, 2009.

26. Sales KJ and Jabbour HN: Cyclooxygenase enzymes and prostaglandins in pathology of the endometrium. Reproduction 126: $559-567,2003$.

27. Prieto L, Quesada JF, Cambero O, Pacheco A, Pellicer A, Codoceo R and Garcia-Velasco JA: Analysis of follicular fluid and serum markers of oxidative stress in women with infertility related to endometriosis. Fertil Steril 98: 126-130, 2012.

28. Kobayashi H, Yamada Y, Kanayama S, Furukawa N, Noguchi T, Haruta S, Yoshida S, Sakata M, Sado T and Oi H: The role of iron in the pathogenesis of endometriosis. Gynecol Endocrinol 25: 39-52, 2009. 
29. Menezo YJ, Silvestris E, Dale B and Elder K: Oxidative stress and alterations in DNA methylation: Two sides of the same coin in reproduction. Reprod Biomed Online 33: 668-683, 2016.

30. Jomova $\mathrm{K}$ and Valko $\mathrm{M}$ : Advances in metal-induced oxidative stress and human disease. Toxicology 283: 65-87, 2011.

31. Verlhac S, Morel M, Bernaudin F, Béchet S, Jung C and Vasile M: Liver iron overload assessment by MRI R2* relaxometry in highly transfused pediatric patients: An agreement and reproducibility study. Diagn Interv Imaging 96: 259-264, 2015.

32. Yoshimoto C, Takahama J, Iwabuchi T, Uchikoshi M, Shigetomi $\mathrm{H}$ and Kobayashi $\mathrm{H}$ : Transverse relaxation rate of cyst fluid can predict malignant transformation of ovarian endometriosis. Magn Reson Med Sci 16: 137-145, 2017.
33. Kawahara N, Yamada Y, Ito F, Hojo W, Iwabuchi $\mathrm{T}$ and Kobayashi H: Discrimination of malignant transformation from benign endometriosis using a near-infrared approach. Exp Ther Med 15: 3000-3005, 2018.

This work is licensed under a Creative Commons Attribution-NonCommercial-NoDerivatives 4.0 International (CC BY-NC-ND 4.0) License. 